\title{
Characterization of Differentially Expressed miRNAs by CXCL12/SDF-1 in Human Bone Marrow Stromal Cells
}

https://doi.org/10.1515/bmc-2021-0015

received July 14, 2021; accepted August 30, 2021.

\begin{abstract}
Stromal cell-derived factor 1 (SDF-1) is known to influence bone marrow stromal cell (BMSC) migration, osteogenic differentiation, and fracture healing. We hypothesize that SDF-1 mediates some of its effects on BMSCs through epigenetic regulation, specifically via microRNAs (miRNAs). MiRNAs are small non-coding RNAs that target specific mRNA and prevent their translation. We performed global miRNA analysis and determined several miRNAs were differentially expressed in response to SDF-1 treatment. Gene Expression Omnibus (GEO) dataset analysis showed that these miRNAs play an important role in osteogenic differentiation and fracture healing. KEGG and GO analysis indicated that SDF-1 dependent miRNAs changes affect multiple cellular pathways, including fatty acid biosynthesis, thyroid hormone signaling, and mucintype O-glycan biosynthesis pathways. Furthermore, bioinformatics analysis showed several miRNAs target genes related to stem cell migration and differentiation. This study's findings indicated that SDF-1 induces some of its effects on BMSCs function through miRNA regulation.
\end{abstract}

\footnotetext{
*Corresponding author: Sadanand Fulzele, Department of Orthopedics, Augusta University, Augusta, GA, E-mail: sfulzele@augusta.edu Matthew L. Potter, Mark Hamrick, Sadanand Fulzele, Department of Orthopedics, Augusta University, Augusta, GA

Sadanand Fulzele, Kathryn Smith, Sagar Vyavahare, Sandeep Kumar, Mark Hamrick, Department of Cell Biology and Anatomy, Augusta University, Augusta, GA

Sudharsan Periyasamy-Thandavan, Georgia Cancer Center, Augusta University, Augusta, GA 30912, United States

Sadanand Fulzele, Mark Hamrick, Carlos M. Isales, Institute of Healthy Aging, Augusta University, Augusta, GA

Sadanand Fulzele, Carlos M. Isales, Departments of Medicine, Augusta University, Augusta, GA

William D. Hill, Department of Pathology and Laboratory Medicine, Medical University of South Carolina, Charleston, SC 29403

William D. Hill, Ralph H Johnson Veterans Affairs Medical Center, Charleston, SC, 29403
}

Keywords: bone marrow stromal cells; SDF-1; miRNA.

\section{Introduction}

Recent developments in regenerative medicine seek to utilize adult stem cells as therapeutics to treat agerelated musculoskeletal disease. Gene reprogramming via manipulating the cellular microenvironment is a promising and unique opportunity to direct adult stem cell fate. Bone marrow stromal cells (BMSCs) are mesenchymal lineage progenitors that give rise to multiple cell types, including osteoblasts, chondrocytes, adipocytes, and other connective tissue cells $[1,2]$. BMSCs are thought to play an important role in the repair and maintenance of various musculoskeletal tissues. The migration and differentiation of adult stem cells, including BMSCs, is a highly regulated by interaction with the cellular milieu. Molecular factors within the milieu, such as growth factors and chemokines, play a vital role in the extracellular regulation of BMSCs [3].

Chemokines are a class of cytokines that induce cell chemotaxis to areas of inflammation. Stromal cell-derived factor 1 (SDF-1), also known as CXCL12, is a chemokine that plays a unique role in the migration of progenitor cell types [4]. SDF-1 mediates its effect primarily through interaction with CXC chemokine receptor-4 (CXCR4) [5]. SDF-1 is known to mediate cell migration, osteogenic differentiation, and cell survival of BMSCs [6-12]. Our group previously reported that SDF-1 accelerates osteogenic differentiation of BMSCs by regulating osteogenic factors (e.g. RUNX2, BMP2, osteocalcin, collagen alpha 1 type 1) [10]. We also reported that SDF-1 promotes BMSCs migration and osteogenic differentiation in vitro, as well as bone formation in vivo [8, 10]. Similarly, other groups found SDF-1 to be implicit during endochondral ossification and fracture healing, specifically the differentiation of BMSCs into hypertrophic chondrocytes [13-15]. Due to the clinical significance of these findings, 
understanding the molecular mechanisms by which SDF-1 exerts its effects on BMSCs proves highly valuable.

Given the ability of SDF-1 to influence various signaling pathways within BMSCs, we postulate that it likely mediates its control via epigenetic regulation [16]. Epigenetic regulation is a mechanism by which gene expression changes occur without changes in genetic makeup [17]. Epigenetic factors, including DNA methylation and miRNAs, are known to be implicit in the differentiation of BMSCs and musculoskeletal development [18-22]. MiRNAs are small non-coding RNAs that bind mRNA at the 3'-untranslated regions (3'UTR). Upon binding, the miRNA prevents translation or promotes degradation of the mRNA, thus negatively regulating gene expression at the post-transcriptional level [23, 24]. Several reports suggest that miRNAs regulate almost all cellular events including cell proliferation, differentiation, and development [25-28]. We hypothesize that SDF-1 induces some of its effects via the regulation of miRNAs. In this study we investigate the SDF-1 dependent regulation of miRNAs within human BMSCs and their correlation with respect to survival, proliferation, migration, and differentiation.

\section{Material and Methods}

Isolation of human BMSCs and SDF-1 treatment: Human bone marrow (BM) from young (18-40 years of age) subjects were obtained under sterile conditions from orthopedic surgery patients as per the Institutional Review Board (IRB) of Augusta University. CD271+ MSCs were extracted from the bone marrow using an isolation kit (Miltenyi Biotec Inc., 130-092-283, Sunnyvale, CA, USA) and washed with a standard culture medium composed of DMEM medium (Corning, 10-013-CM, Corning, NY, USA), $1 \%$ antibiotics-antimycotics (AA; Invitrogen, 15240062, Carlsbad, CA, USA) and 10\% fetal bovine serum (FBS). Cells were transferred to $100 \mathrm{~mm}$ culture dish and incubated at $37^{\circ} \mathrm{C}$ and $5 \%$ carbon dioxide (CO2) in a humidified environment. After $24 \mathrm{~h}$, the media with nonadherent cells was removed. The adherent cells were washed in phosphate buffer saline (PBS) and further expanded by incubation in the fresh standard culture medium. Culture-expanded CD271+ BMSCs of passage 1 were used for treating with the SDF-1. Human BMSCs were cultured on 24 well plates treated with or without SDF-1 (50 $\mathrm{ng} / \mathrm{mL}$ concentration) for $72 \mathrm{hrs}$. Media was changed every day with or without SDF-1. At the end of 72 hrs, miRNAs were isolated using a miRNA isolation kit (SABiosciences Corporation, Frederick, MD, USA).
Informed consent: Informed consent has been obtained from all individuals included in this study.

Ethical approval: The research related to human use has been complied with all the relevant national. regulations, institutional policies and in accordance the tenets of the Helsinki Declaration, and has been approved by the Institutional Review Board (IRB) of Augusta University.

MicroRNA Array and Bioinformatics Analysis; Total miRNA isolation was performed using a miRNA isolation kit (SABiosciences Corporation, Frederick, MD, USA) that captures small RNAs with lengths less than 200 nucleotides. RNA concentrations were determined using a NanoDrop 1000 Spectrophotometer (NanoDrop Technologies, Wilmington, DE, USA). The quality of RNA samples was characterized by an Agilent BioAnalyzer (Agilent Technologies, Santa Clara, CA, USA) using an RNA6000 Nano Chip (Agilent). miRNA microarrays were performed using an Affymetrix GeneChip ${ }^{\circledR}$ miRNA 4.0 array at the Integrated Genomics Core, Augusta University, GA, USA. The miRNA profile was analyzed for the hierarchical clustering of miRNA to generate heat maps and principal component analysis (PCA). T-tests were conducted to calculate the $\mathrm{p}$-value and determine whether miRNA levels were significantly changed in SDF-1 treatment versus control groups. Gene Ontology (GO) enrichment analysis and Kyoto Encyclopedia of Genes and Genomes (KEGG) pathway annotation were performed on differentially expressed miRNAs using DIANA-miRPath v.3. Bioinformatics software (http://www.targetscan. org/vert_72/ and http://www.mirdb.org/) were used to predict gene targets of differentially expressed miRNAs considered to be novel.

GEO Database Analysis of Differentially Expressed MiRNA; Tocorrelate our microarray results, we performedan analysis of existing microarray data in the Gene Expression Omnibus (GEO) public repository. GEO allows researchers to archive and distribute high throughput gene sequencing and microarray data sets [29]. Users can analyze genomic expression profiles of interest from previously performed pre-clinical studies. We searched the GEO database for microarray datasets of miRNA differentially expressed under three unique criteria. 1) GEO was queried for datasets of fracture healing models. This search yielded the study by Hadjiargyrou et al. (GEO accession GSE76197), which profiled miRNA in murine femur fracture across 14 days post-fracture versus intact control. 2) GEO was queried for datasets from osteogenic differentiation of mesenchymal stem cells or bone marrow stromal cells. Only datasets with significant results were included for analysis. The search yielded the following datasets: GSE159508, GSE134946 
, GSE72429, GSE115197. 3) GEO was queried for datasets derived from BMP2 osteogenic growth factor treatment of cell lines derived from mesenchymal origin. The search yielded a dataset by Bae et al. (GEO accession GSE37036) showcasing miRNA expression in $\mathrm{C} 2 \mathrm{C} 12$ myoblasts treated with BMP2 for 72 hours.

GEO2R interactive tool was used to determine differential expression between treatment and control groups by calculating log base 2 of fold change $(\log 2(\mathrm{FC})$ ). Significance in fold change was determined from the adjusted p-value parameter calculated by the GEO2R interactive tool. The Benjamini \& Hochberg false discovery rate method for $p$-value adjustment was selected as it is the most commonly used adjustment for microarray data. It provides a balance between the discovery of statistically significant genes and the limitation of false positives.

\section{Results}

MiRNA Differentially Expressed Following SDF-1 Treatment: miRNA microarray analysis was conducted to compare miRNA profiles of human BMSCs with and without SDF-1 treatment. We found 104 miRNAs to be differentially regulated $(\mathrm{p}<0.05)$ with an absolute fold change of 1.5 or greater following SDF-1 treatment. Out of these 104 miRNAs, 49 miRNAs were downregulated, and 55 miRNAs were upregulated. Table 1 presents the top 50 most differentially expressed miRNAs. A heat map with hierarchical clustering visually depicts the unique profiles of miRNA from SDF-1 treated and control groups (Figure 1).

Principal Component Analysis; Principle component analysis (PCA) was performed to distinguish the miRNA profile in SDF-1 treated versus control BMSCs. The PCA depicts unique clusters for treated samples versus control samples highlighting the clear distinction between these two groups (Figure 2).

Novel miRNAs Target Key Genes in Migration and Differentiation; In silico analysis was performed on novel miRNAs with lesser-known functions scarcely reported in the existing literature. To elucidate their roles, miRNAs underwent bioinformatic analysis utilizing targetscan.org and mirdb.org. Criteria for target genes include complementary binding between miRNA seed sequence and the mRNA transcript for the gene of interest. Several target genes were identified that are known to modulate stem cell migration and differentiation into musculoskeletal tissues. Table 2 presents these novel miRNAs and their respective gene targets.
Table 1: Select (top 50) differentially expressed miRNAs in response to SDF-1 treatment in human BMSCs.

\begin{tabular}{|c|c|c|}
\hline MicroRNA ID/Probeset ID & Fold-Change & $p$-Value \\
\hline hsa-miR-654-5p & -2.5901 & 0.02547 \\
\hline hsa-miR-339-3p & -2.25636 & 0.02332 \\
\hline hsa-miR-1226-5p & -2.14647 & 0.00692 \\
\hline hsa-miR-23b-5p & -2.11548 & 0.02960 \\
\hline hsa-miR-330-3p & -2.07703 & 0.01226 \\
\hline hsa-miR-668-5p & -2.06023 & 0.00677 \\
\hline hsa-miR-3162-5p & -1.99216 & 0.02709 \\
\hline hsa-miR-3911 & -1.94415 & 0.02799 \\
\hline hsa-miR-6831-5p & -1.8794 & 0.01496 \\
\hline hsa-miR-92b-5p & -1.87062 & 0.00466 \\
\hline hsa-mir-4673 & -1.83306 & 0.02000 \\
\hline hsa-miR-493-3p & -1.83031 & 0.01104 \\
\hline hsa-miR-1233-5p & -1.81938 & 0.00178 \\
\hline hsa-miR-27a-5p & -1.78655 & 0.00742 \\
\hline hsa-miR-34c-3p & -1.77045 & 0.02972 \\
\hline hsa-miR-1275 & -1.76887 & 0.00589 \\
\hline hsa-miR-1268b & -1.70929 & 0.01389 \\
\hline hsa-miR-4484 & -1.69334 & 0.00523 \\
\hline hsa-miR-4640-5p & -1.67491 & 0.01385 \\
\hline hsa-miR-423-5p & -1.66952 & 0.00188 \\
\hline hsa-miR-23a-5p & -1.63963 & 0.00454 \\
\hline hsa-miR-134-5p & -1.6315 & 0.00105 \\
\hline hsa-miR-550a-3-5p & -1.58899 & 0.01087 \\
\hline hsa-miR-324-5p & -1.58876 & 0.01565 \\
\hline hsa-miR-6850-5p & -1.58559 & 0.00621 \\
\hline hsa-miR-376a-3p & 1.76442 & 0.01016 \\
\hline hsa-miR-146b-5p & 1.77157 & 0.01273 \\
\hline hsa-mir-548q & 1.77686 & 0.02127 \\
\hline hsa-miR-940 & 1.8313 & 0.01507 \\
\hline hsa-miR-505-5p & 1.88502 & 0.03045 \\
\hline hsa-miR-4688 & 1.88885 & 0.00777 \\
\hline hsa-miR-6769a-5p & 1.92262 & 0.00585 \\
\hline hsa-miR-151b & 2.00276 & 0.01503 \\
\hline hsa-miR-4284 & 2.02122 & 0.04587 \\
\hline hsa-miR-148a-3p & 2.04888 & 0.01323 \\
\hline hsa-let-7f-5p & 2.05026 & 0.00014 \\
\hline hsa-miR-7845-5p & 2.12776 & 0.03009 \\
\hline hsa-miR-6891-5p & 2.14821 & 0.00195 \\
\hline hsa-miR-493-5p & 2.17137 & 0.00349 \\
\hline hsa-let-7d-3p & 2.19535 & 0.04102 \\
\hline hsa-miR-503-5p & 2.20447 & 0.01386 \\
\hline hsa-miR-3121-3p & 2.27425 & 0.00499 \\
\hline hsa-miR-495-3p & 2.36848 & 0.00776 \\
\hline hsa-miR-30b-5p & 2.43776 & 0.03964 \\
\hline hsa-miR-376c-3p & 2.47408 & 0.00670 \\
\hline hsa-miR-140-5p & 2.48671 & 0.00463 \\
\hline hsa-miR-196a-5p & 2.68803 & 0.02805 \\
\hline hsa-miR-1246 & 2.74544 & 0.01874 \\
\hline hsa-miR-4706 & 2.96761 & 0.01040 \\
\hline hsa-miR-24-2-5p & 3.12261 & 0.00626 \\
\hline
\end{tabular}




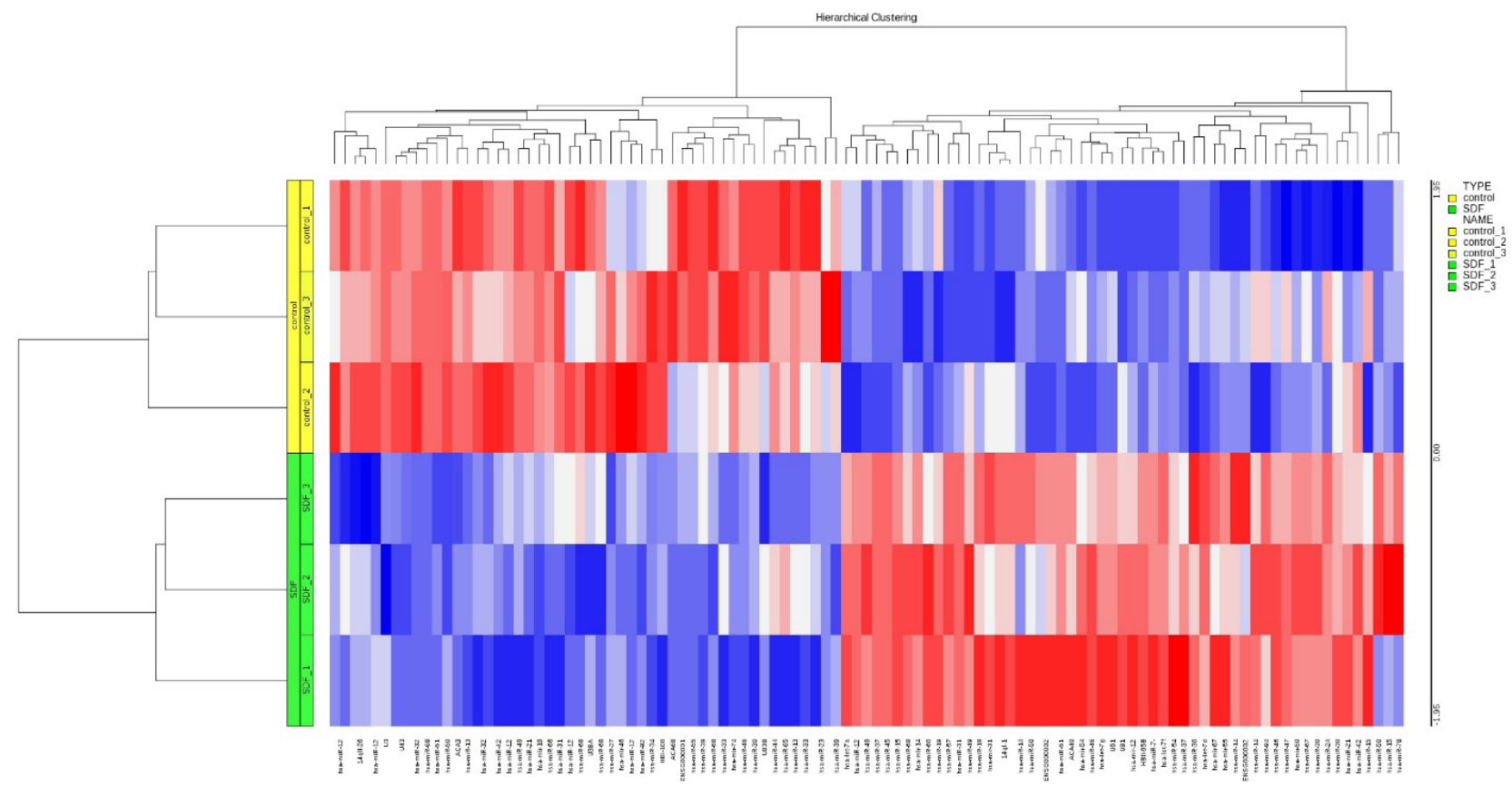

Figure 1: Hierarchical clustered heat map depicting miRNA expression in SDF-1 treated human BMSCs versus control ( $n=3$ each group). Differential expression pattern is observed between the miRNAs of the two groups.

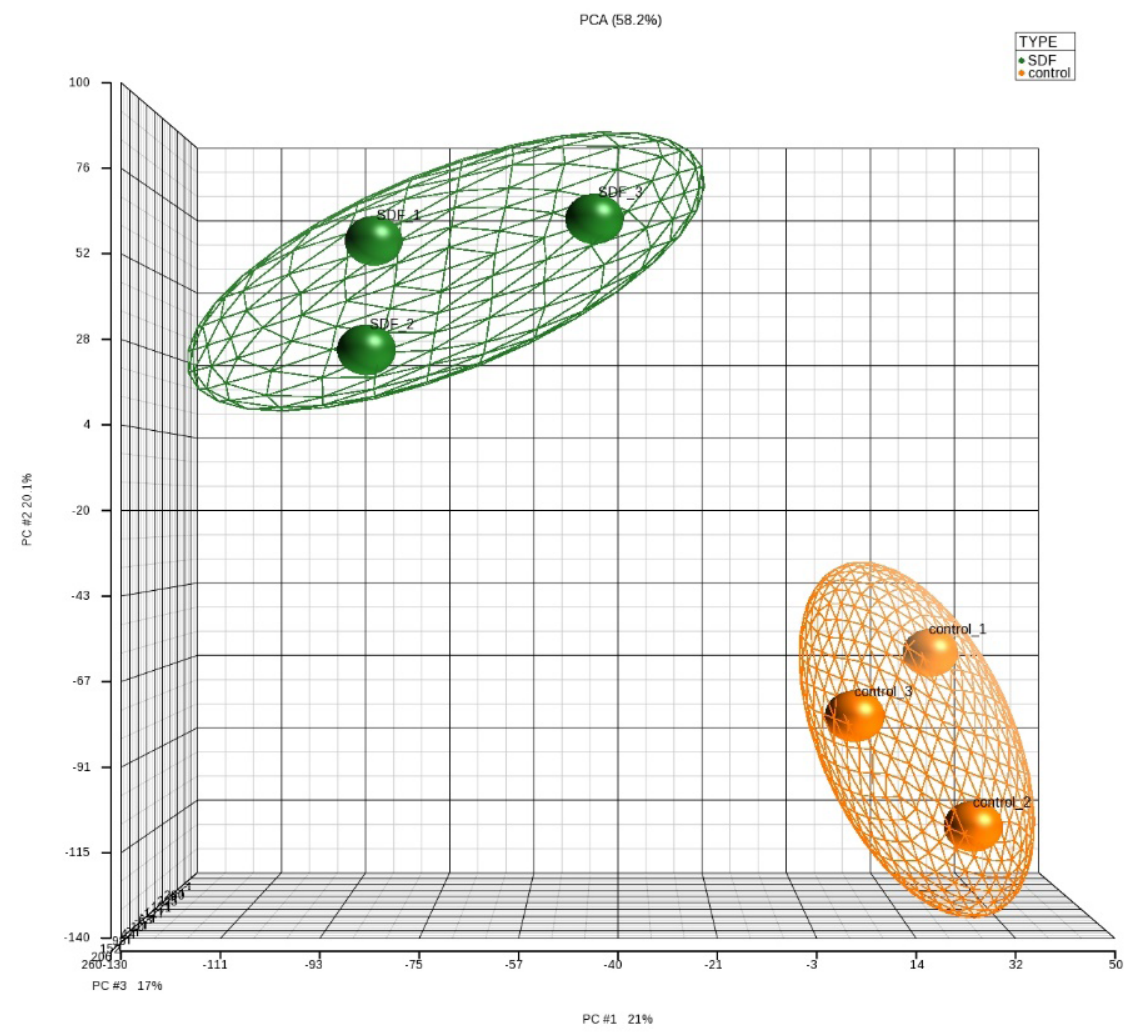

Figure 2: Primary component analysis mappings of SDF-1 treated human BMSCs versus control. SDF-1 treatment and control group show a unique miRNA clustering pattern for each group. Differential expression pattern confirms that seen in miRNA heat map. 
Table 2: Target genes of differentially expressed miRNA in response to SDF-1 treatment in human BMSCs.

\begin{tabular}{|c|c|c|c|}
\hline \multirow[t]{2}{*}{ microRNA } & \multicolumn{2}{|l|}{ No of Targets } & \multirow{2}{*}{$\begin{array}{l}\text { Stem Cell Related Genes } \\
\text { Common Targets }\end{array}$} \\
\hline & Target Scan & miRDB Scan & \\
\hline hsa-miR-1226-5p & 3601 & 304 & $\begin{array}{l}\text { BMPER, BMP2, COLGALT2, QSOX1, FOXN3, FOXD4L3, TGFA, IL18, ITGA10, } \\
\text { ITGAL, ITGB2, DLX3, WNT7A, SMURF1 }\end{array}$ \\
\hline hsa-miR-3911 & 3759 & 496 & $\begin{array}{l}\text { BMP4, COL8A1, COLGALT1, FOXP3, IL1RAP, IL17RA, IL6ST, ITGB3, CCR3, } \\
\text { WNT7A, RHOG, FGF11 }\end{array}$ \\
\hline hsa-miR-4640-5p & 4689 & 727 & $\begin{array}{l}\text { COL26A1, COL4A3BP, FOXR2, FOXA3, FOXL2, TNFRSF12A, TNFRSF18, } \\
\text { C1QTNF8, TGFB3, IL10RA, IL15, IL2RG, ILF3, ITGA2, ITGA5, TRAF4, WNT5B, } \\
\text { WNT7B, RHOA, RHOF, MAPK4, MAPKAPK3, MAPK14, MAPK15, MAPK8IP3, } \\
\text { MAPKBP1 }\end{array}$ \\
\hline hsa-miR-4706 & 1615 & 86 & SOX11, FOXRED2, FOXE1, HOXC10, TNFRSF8, IL17REL, CXCR3 \\
\hline hsa-miR-7845-5p & 3726 & 387 & SOX4, SOX12, FOXI2, HOXC4, TNFAIP3, IL9R, ITGA6, TAB2 \\
\hline hsa-miR-6769a-5p & 4673 & 431 & $\begin{array}{l}\text { BMP6, COL4A5, COL6A5, FOXP4, HOXB9, HOXA11, HOXB13, TNFRSF21, ILF3, } \\
\text { ITGB2, WNT7B, WNT4, RHOF }\end{array}$ \\
\hline
\end{tabular}

Table 3: KEGG annotation of cellular pathways potentially affected by downregulated miRNAs (a) and upregulated miRNAs (b) due to SDF-1 treatment in human BMSCs.

\begin{tabular}{|c|c|c|c|}
\hline \multicolumn{4}{|l|}{ (a) } \\
\hline KEGG Pathway & $p$-Value & No. of Genes Involved & No. of miRNAs Involved \\
\hline Fatty acid biosynthesis & $6.50 \mathrm{E}-09$ & 2 & 2 \\
\hline Tyrosine metabolism & 0.04460 & 10 & 7 \\
\hline Other types of 0-glycan biosynthesis & 0.04460 & 11 & 10 \\
\hline Thyroid hormone signaling pathway & 0.04460 & 34 & 13 \\
\hline \multicolumn{4}{|l|}{ (b) } \\
\hline KEGG Pathway & $p$-Value & No. of Genes Involved & No. of miRNAs Involved \\
\hline Prion diseases & 8.70E-19 & 3 & 3 \\
\hline GABAergic synapse & 4.30E-09 & 16 & 8 \\
\hline Nicotine addiction & $9.79 \mathrm{E}-06$ & 11 & 6 \\
\hline Biotin metabolism & $9.37 \mathrm{E}-05$ & 1 & 2 \\
\hline Morphine addiction & 0.00067 & 16 & 7 \\
\hline Mucin type 0-Glycan biosynthesis & 0.00146 & 6 & 5 \\
\hline Choline metabolism in cancer & 0.00296 & 21 & 13 \\
\hline Sulfur metabolism & 0.02884 & 3 & 3 \\
\hline
\end{tabular}

KEGG Pathway Annotation and GO Enrichment Analysis of Differentially Expressed MiRNAs; The collective effect of differentially expressed miRNA was characterized with Kyoto Encyclopedia of Genes and Genomes (KEGG) pathway annotation and Gene Ontology (GO) enrichment analysis. KEGG annotation identified several cellular pathways that are affected by these miRNAs (Table 3). Notable pathways include fatty acid biosynthesis, thyroid hormone signaling, and mucin-type O-glycan biosynthesis pathways. GO analysis determined 102 biological processes associated with the differentially expressed miRNAs (Table 4). Common processes affected by both upregulated and downregulated miRNAs include gene expression, nucleic acid binding transcription factor activity, cellular protein modification process, enzyme binding, and small molecule metabolic process. 
Table 4: GO analysis of biological processes (top 50) potentially affected by downregulated miRNAs (a) and upregulated miRNAs (b) due to SDF-1 treatment in human BMSCs.

\begin{tabular}{|c|c|c|c|}
\hline GO Category & $p$-Value & Genes & miRNAs \\
\hline organelle & $3.09 \mathrm{E}-80$ & 2037 & 20 \\
\hline ion binding & $2.08 \mathrm{E}-59$ & 1318 & 20 \\
\hline cellular nitrogen compound metabolic process & $1.72 \mathrm{E}-44$ & 1000 & 20 \\
\hline biosynthetic process & $2.83 \mathrm{E}-30$ & 842 & 20 \\
\hline small molecule metabolic process & $4.38 \mathrm{E}-25$ & 519 & 20 \\
\hline blood coagulation & $1.23 \mathrm{E}-15$ & 120 & 19 \\
\hline cellular protein modification process & $1.23 \mathrm{E}-15$ & 475 & 20 \\
\hline catabolic process & $2.19 \mathrm{E}-15$ & 413 & 20 \\
\hline gene expression & $2.23 \mathrm{E}-15$ & 133 & 19 \\
\hline neurotrophin TRK receptor signaling pathway & $1.22 \mathrm{E}-14$ & 70 & 15 \\
\hline symbiosis, encompassing mutualism through parasitism & $1.38 \mathrm{E}-12$ & 121 & 18 \\
\hline cellular protein metabolic process & $2.43 \mathrm{E}-12$ & 108 & 16 \\
\hline molecular function & $7.74 \mathrm{E}-12$ & 3342 & 20 \\
\hline cytoskeletal protein binding & $1.13 \mathrm{E}-11$ & 186 & 20 \\
\hline enzyme binding & $1.68 \mathrm{E}-11$ & 280 & 19 \\
\hline cellular component & $3.94 \mathrm{E}-11$ & 3380 & 20 \\
\hline viral process & $8.06 \mathrm{E}-11$ & 104 & 17 \\
\hline nucleobase-containing compound catabolic process & $3.91 \mathrm{E}-10$ & 197 & 20 \\
\hline membrane organization & $5.65 \mathrm{E}-09$ & 132 & 19 \\
\hline protein complex & 2.67E-08 & 735 & 20 \\
\hline glycosaminoglycan metabolic process & 4.57E-08 & 34 & 14 \\
\hline protein binding transcription factor activity & $5.32 \mathrm{E}-08$ & 110 & 18 \\
\hline transmembrane transporter activity & $2.99 \mathrm{E}-07$ & 231 & 20 \\
\hline Fc-epsilon receptor signaling pathway & 4.34E-07 & 38 & 13 \\
\hline vesicle-mediated transport & $1.53 \mathrm{E}-06$ & 229 & 18 \\
\hline nucleic acid binding transcription factor activity & $1.99 \mathrm{E}-06$ & 198 & 19 \\
\hline activation of signaling protein activity involved in unfolded protein response & $3.04 \mathrm{E}-06$ & 22 & 13 \\
\hline platelet degranulation & $3.04 \mathrm{E}-06$ & 24 & 13 \\
\hline platelet activation & $1.25 \mathrm{E}-05$ & 49 & 14 \\
\hline cell-cell signaling & $2.48 \mathrm{E}-05$ & 139 & 19 \\
\hline cytosol & $3.63 \mathrm{E}-05$ & 520 & 20 \\
\hline synaptic transmission & $5.02 \mathrm{E}-05$ & 91 & 18 \\
\hline toll-like receptor TLR1:TLR2 signaling pathway & $5.11 \mathrm{E}-05$ & 19 & 8 \\
\hline toll-like receptor TLR6:TLR2 signaling pathway & $5.11 \mathrm{E}-05$ & 19 & 8 \\
\hline cellular component assembly & $6.83 \mathrm{E}-05$ & 244 & 20 \\
\hline macromolecular complex assembly & 0.0001746 & 168 & 19 \\
\hline vitamin metabolic process & 0.0002525 & 21 & 12 \\
\hline toll-like receptor 10 signaling pathway & 0.0003075 & 17 & 6 \\
\hline extracellular matrix disassembly & 0.0003484 & 28 & 14 \\
\hline post-translational protein modification & 0.0003484 & 35 & 14 \\
\hline mRNA metabolic process & 0.0003484 & 44 & 16 \\
\hline enzyme regulator activity & 0.0003484 & 164 & 19 \\
\hline response to stress & 0.0003484 & 414 & 20 \\
\hline leukocyte migration & 0.0003586 & 31 & 12 \\
\hline extracellular matrix organization & 0.0003644 & 84 & 18 \\
\hline water-soluble vitamin metabolic process & 0.0003887 & 19 & 12 \\
\hline platelet alpha granule lumen & 0.0004625 & 14 & 8 \\
\hline inositol phosphate metabolic process & 0.0007525 & 14 & 8 \\
\hline cell death & 0.0007856 & 175 & 19 \\
\hline chondroitin sulfate metabolic process & 0.0007939 & 15 & 10 \\
\hline
\end{tabular}


Table 4: GO analysis of biological processes (top 50) potentially affected by downregulated miRNAs (a) and upregulated miRNAs (b) due to SDF-1 treatment in human BMSCs.

(b)

\begin{tabular}{|c|c|c|c|}
\hline GO Category & $p$-Value & Genes & miRNAs \\
\hline organelle & $1.132 \mathrm{E}-41$ & 1034 & 20 \\
\hline cellular nitrogen compound metabolic process & $4.30 \mathrm{E}-27$ & 526 & 19 \\
\hline ion binding & $1.465 \mathrm{E}-25$ & 650 & 19 \\
\hline biosynthetic process & $2.089 \mathrm{E}-12$ & 414 & 19 \\
\hline small molecule metabolic process & $1.419 \mathrm{E}-08$ & 247 & 20 \\
\hline cellular protein modification process & $7.94 \mathrm{E}-08$ & 242 & 19 \\
\hline gene expression & $1.065 \mathrm{E}-07$ & 69 & 16 \\
\hline molecular function & $2.308 \mathrm{E}-07$ & 1688 & 20 \\
\hline cellular component & $2.40 \mathrm{E}-07$ & 1709 & 20 \\
\hline neurotrophin TRK receptor signaling pathway & $1.807 \mathrm{E}-06$ & 35 & 14 \\
\hline catabolic process & $1.807 \mathrm{E}-06$ & 204 & 18 \\
\hline blood coagulation & $2.374 \mathrm{E}-06$ & 58 & 17 \\
\hline protein complex & $2.374 \mathrm{E}-06$ & 389 & 19 \\
\hline toll-like receptor TLR1:TLR2 signaling pathway & 0.000331 & 13 & 8 \\
\hline toll-like receptor TLR6:TLR2 signaling pathway & 0.000331 & 13 & 8 \\
\hline nucleobase-containing compound catabolic process & 0.000415 & 97 & 17 \\
\hline cell-cell signaling & 0.000424 & 78 & 19 \\
\hline enzyme binding & 0.000559 & 134 & 18 \\
\hline nucleoplasm & 0.000570 & 126 & 18 \\
\hline cellular protein metabolic process & 0.000677 & 49 & 14 \\
\hline platelet activation & 0.001002 & 28 & 13 \\
\hline mitotic cell cycle & 0.001150 & 43 & 14 \\
\hline viral process & 0.001360 & 48 & 13 \\
\hline nucleic acid binding transcription factor activity & 0.001360 & 103 & 17 \\
\hline post-translational protein modification & 0.001411 & 22 & 11 \\
\hline response to stress & 0.001583 & 222 & 19 \\
\hline toll-like receptor signaling pathway & 0.002292 & 18 & 9 \\
\hline synaptic transmission & 0.002292 & 50 & 15 \\
\hline pyrimidine nucleoside biosynthetic process & 0.002340 & 4 & 2 \\
\hline toll-like receptor 4 signaling pathway & 0.002396 & 16 & 8 \\
\hline toll-like receptor 10 signaling pathway & 0.002454 & 11 & 7 \\
\hline apoptotic signaling pathway & 0.002901 & 22 & 12 \\
\hline symbiosis, encompassing mutualism through parasitism & 0.002901 & 52 & 13 \\
\hline Fc-epsilon receptor signaling pathway & 0.003500 & 19 & 11 \\
\hline cell death & 0.004487 & 96 & 14 \\
\hline toll-like receptor 2 signaling pathway & 0.004505 & 13 & 8 \\
\hline toll-like receptor 5 signaling pathway & 0.007802 & 11 & 7 \\
\hline TRIF-dependent toll-like receptor signaling pathway & 0.009640 & 11 & 7 \\
\hline Fc-gamma receptor signaling pathway involved in phagocytosis & 0.010326 & 11 & 6 \\
\hline epidermal growth factor receptor signaling pathway & 0.010326 & 26 & 12 \\
\hline membrane organization & 0.010326 & 60 & 14 \\
\hline glycosaminoglycan metabolic process & 0.012232 & 15 & 11 \\
\hline transmembrane transporter activity & 0.012232 & 111 & 18 \\
\hline anatomical structure morphogenesis & 0.014649 & 17 & 9 \\
\hline toll-like receptor 9 signaling pathway & 0.015862 & 11 & 6 \\
\hline carnitine shuttle & 0.018310 & 4 & 4 \\
\hline MyD88-independent toll-like receptor signaling pathway & 0.022856 & 11 & 7 \\
\hline transcription initiation from RNA polymerase II promoter & 0.027497 & 28 & 12 \\
\hline MyD88-dependent toll-like receptor signaling pathway & 0.027508 & 14 & 9 \\
\hline regulation of transcription from RNA polymerase II promoter in response to hypoxia & 0.030148 & 6 & 5 \\
\hline
\end{tabular}



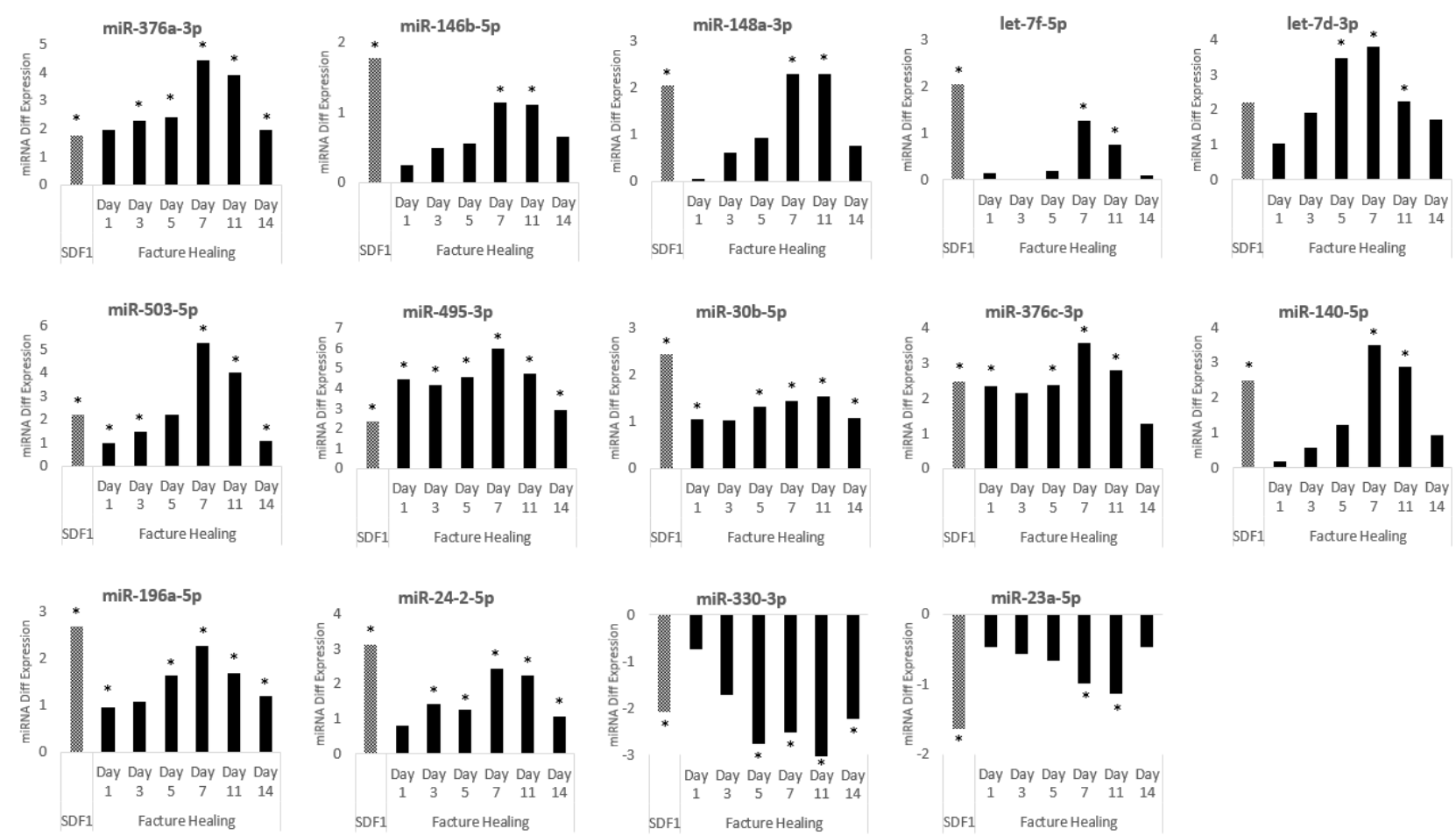

Figure 3: Common miRNAs regulated in presence of SDF-1 treatment (at 72 hours) and during murine femur fracture healing (across multiple time points ranging from day 1 through day 14). Fracture healing data was retrieved from GEO dataset uploaded by Hadjiargyrou et al. (GEO accession GSE76197) in which murine bone fractures were generated and miRNA-enriched RNA was isolated from calluses at post-fracture days $1,3,5,7,11$, and 14 and compared to intact bone (control) $(n=3)$. Microarray analysis revealed 306 and 374 up- and down-regulated miRNAs, respectively, of which 14 were similar to our study and presented in this figure with their respective fold changes. Significance in fold change ${ }^{*}$ ) of GEO data was determined by GEO2R adjusted $p$-value $<0.05$.

SDF-1 Versus Fracture Healing and Osteogenic Induced MiRNA Expression Profile; GEO dataset archived by Hadjiargyrou et al. (GEO accession GSE76197) was analyzed to determine the differential expression of miRNA in murine femur fracture model across 14 days, and results were compared to that of SDF-1. The analysis determined 12 common miRNAs were significantly upregulated in our study and fracture healing [Hadjiargyrou et al., 2016)] at one or more time points across the 14-day study. Conversely, two common miRNAs were significantly downregulated across both studies. These specific miRNAs are presented in Figure 3. Subsequent GEO search yielded several more datasets depicting differently regulated miRNAs during osteogenic differentiation of human mesenchymal stem/stromal cells (GSE159508, GSE134946, GSE72429, GSE115197). The analysis determined that several common miRNAs were differentially expressed across these studies and with SDF-1 treatment. These results are presented in Table 5.

SDF-1 Versus BMP2 Induced MiRNA Expression Profile; GEO dataset archived by Bae et al. (GEO accession
GSE37036) was analyzed to determine the miRNA expression profile with BMP2 treatment, and results were compared to that of SDF-1. The analysis revealed several common miRNAs were differentially expressed in both studies. Specifically, let-7f-5p, miR-140-5p, miR-196a-5p, and miR-24-2-5p were upregulated in both SDF-1 and BMP2 treatment, as presented in Figure 4. miR-330-3p was downregulated with the treatment of either growth factor. This suggests that both BMP2 and SDF-1 induce osteogenic differentiation by regulating the same downstream osteogenic miRNAs. Furthermore, SDF-1 is known to have potential BMP2 induced bone formation, a phenomenon that could be explained in part by shared downstream osteogenic miRNA.

\section{Discussion}

Adult bone marrow stromal cells are the primary source of stem cells in the field of regenerative medicine. As mentioned previously, the extracellular milieu plays 


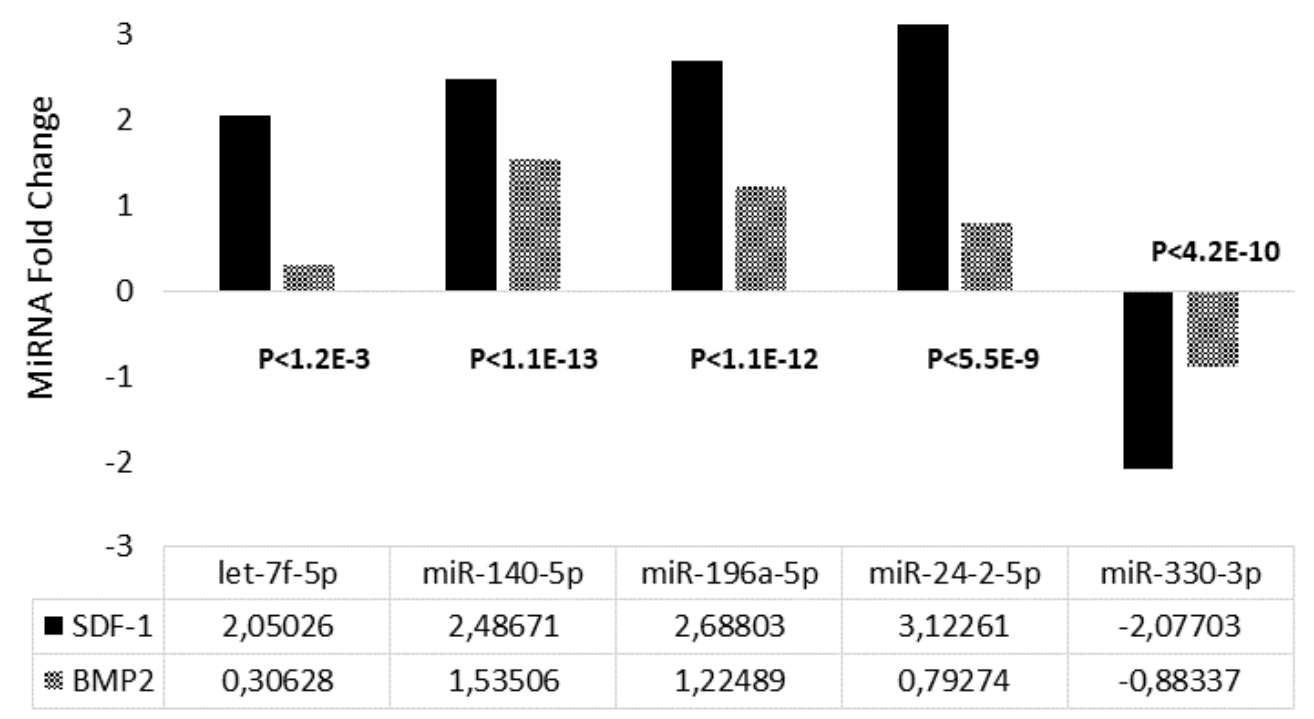

Figure 4: Common miRNAs regulated in the presence of SDF-1 treatment (at 72 hours) and BMP2 treatment (at 72 hours). BMP2 data was determined from GEO database analysis of dataset by Bae et al. (GEO accession GSE37036) in which C2C12 cells were treated with BMP2 $(300 \mathrm{ng} / \mathrm{mL})$ or vehicle (control) for 72 hours $(n=2)$. Afterwards miRNA was isolated and Exiqon miRNA microarray was performed yielding a total of 34 differentially expressed miRNAs, of which five common miRNAs showed similar trend in the presence of SDF-1 and BMP2 treatment. Reported $p$-values are adjusted values calculated by GEO2R analyzer that indicate significance in the fold change (differential expression) for BMP2 treatment versus control.

a paramount role in controlling the migration and differentiation of BMSCs. Manipulating this environment offers a unique opportunity to control cell fate and achieve enhanced therapeutic results. Based on previous findings from our group and others, extracellular SDF-1 is beneficial for osteogenesis and fracture healing [8-10, 13-15]. We hypothesized that extracellular SDF-1 mediates its effects on BMSCs via miRNA-dependent gene regulation.

While various studies have shown the pervasive role of miRNAs regulating cellular events, no study has previously identified SDF-1 regulation of miRNAs in human BMSCs. Our identification of these miRNAs provides an important link in understanding the mechanism by which SDF-1 exerts its osteogenic effect on BMSCs. Herein we identified a list of miRNAs that were differentially expressed in BMSCs following SDF-1 treatment. Several of these miRNAs are also regulated in osteogenic differentiation and fracture healing. Comparing our findings with the GEO dataset by Hadjiargyrou et al. (in which they analyzed miRNA expression in murine femur fracture) 14 common miRNAs were expressed similarly across both studies (Figure 3). Furthermore, we identified several common miRNAs regulated in the presence of SDF-1 and during osteogenic differentiation of mesenchymal stem/stromal cells (Table 5). We speculate that SDF-1 partially enhances fracture healing and osteogenic differentiation through the regulation of these miRNAs.

Several groups have demonstrated SDF-1 potentiates BMP2 induced bone formation [10, 30, 31]. BMP2 is known to enhance the proliferation and osteogenic differentiation of BMSCs [32-34]. Comparing our findings with the GEO dataset by Bae et al. (in which miRNA expression was analyzed in cells treated with BMP2) several common miRNAs were differentially expressed across both studies (Figure 4). These findings suggest SDF-1 and BMP2 could act synergistically via shared downstream osteogenic miRNA. Additionally, our results show SDF-1 downregulates miR-654-5p, a miRNA known to directly target BMP2 within BMSCs (verified by reporter assay) [35]. MiR-654-5p is also observed to be persistently decreased in patients during osteoblast differentiation [36]. Taken together, this is consistent with our prior finding that SDF-1 increases BMP2 mRNA levels in vitro [10]. BMP2 is approved by the FDA for lumbar fusion surgery and is used off-label in many other applications, despite concerns of pro-oncogenic effects [37]. Our findings support the use of lower BMP2 dosage in conjunction with synergistic molecules to achieve clinical outcomes with decreased oncogenic risk. 
Table 5: List of common miRNAs differently expressed after SDF-1 treatment and after various osteogenic differentiation protocols (retrieved via GEO database analysis). GSE159508 presents human periodontal ligament stem cells (hPDLSCs) cultured in osteogenic medium for 14 days compared to hPDLSCs cultured in normal medium (10\% FBS in DMEM) for 14 days. GSE134946 and GSE115197 presents human BMSCs cultured in osteogenic medium for 7 days compared to non-induced BMSCs from day 0. GSE72429 presents human synovial membrane MSCs and adipose derived stem cells (ADSCs) osteogenically differentiated compared to undifferentiated controls. Differential expression is reported as miRNA fold change after treatment compared to respective control. All reported fold changes are statistically significant based upon GEO2R adjusted $p$-value.

\begin{tabular}{|c|c|c|c|c|c|c|}
\hline \multirow[t]{2}{*}{ MicroRNA ID } & \multirow{2}{*}{$\begin{array}{l}\text { SDF-1 Treatment } \\
(n=3)\end{array}$} & \multicolumn{5}{|c|}{ GEO Accession ID } \\
\hline & & $\begin{array}{l}\text { GSE159508 }(n \\
=3)\end{array}$ & $\begin{array}{l}\text { GSE134946 } \\
(n=3)\end{array}$ & $\begin{array}{l}\text { GSE72429* } \\
(n=3)\end{array}$ & $\begin{array}{l}\text { GSE72429** } \\
(n=2)\end{array}$ & $\begin{array}{l}\text { GSE115197 } \\
(n=2)\end{array}$ \\
\hline miR-654-5p & -2.59 & - & -2.95 & - & - & - \\
\hline miR-339-3p & -2.26 & - & -3.03 & - & -2.10 & - \\
\hline miR-493-3p & -1.83 & - & -3.95 & - & - & - \\
\hline miR-27a-5p & -1.79 & -1.26 & - & - & - & - \\
\hline $\operatorname{miR}-146 b-5 p$ & 1.77 & 2.44 & - & - & 9.25 & 0.01 \\
\hline mir-548q & 1.78 & 1.39 & - & 7.29 & - & - \\
\hline miR-940 & 1.83 & 1.59 & 1.32 & 0.86 & - & - \\
\hline miR-505-5p & 1.89 & 0.70 & - & - & - & - \\
\hline miR-4284 & 2.02 & - & - & 1.14 & - & - \\
\hline miR-148a-3p & 2.05 & 1.06 & - & - & - & - \\
\hline let-7d-3p & 2.20 & 1.28 & - & - & - & - \\
\hline miR-24-2-5p & 3.12 & - & - & 0.25 & - & - \\
\hline
\end{tabular}

* Synovial membrane MSC samples, osteogenic differentiation versus undifferentiated control

** Adipose derived stem cell samples, osteogenic differentiation versus adipogenic differentiation (control)

Existing literature indicates that several differentially expressed miRNAs by SDF1 play an important role in osteogenic differentiation of BMSCs. MiR-339 has been found to inhibit the osteogenic differentiation of BMSC via the direct targeting of DLX5, a known factor in osteogenic differentiation [38]. We speculate that SDF-1 likely enhances BMSC osteogenesis via the downregulation of miR-339-3p. MiR-503-5p has been found to promote bone formation by targeting the negative osteogenic regulator SMURF1 within BMSCs [39]. Our results suggest SDF-1 decreases SMURF1 by upregulating miR-503-3p. Let$7 \mathrm{f}-5 \mathrm{p}$ has been found to promote cell survival in various cell lines by targeting caspase- 3 and caspase- 9 while increasing levels of anti-apoptotic factors Bcl-2 and Bcl-xL $[40,41]$. Taken with our results, this indicates an increase in cell survival of BMSCs mediated by SDF-1, consistent with previous reports [9]. We also identified several novel miRNAs with no prior known functions. Bioinformatic analysis revealed a considerable number of these miRNAs are involved in stem cell homing and commitment to musculoskeletal lineage (Table 2).
To better understand the collective effect of differentially expressed miRNA, KEGG pathway annotation and GO enrichment analysis was conducted. KEGG annotation identified several cellular pathways potentially affected, with fatty acid biosynthesis, thyroid hormone signaling, and mucin-type O-glycan biosynthesis pathways most relevant. Fatty acids and their metabolites have been well linked to stem cell proliferation and differentiation [42]. Both saturated and unsaturated fatty acids have differential effects on BMSC survival [43]. Furthermore, polyunsaturated fatty acids have been found to modulate proliferation, migration, and differentiation of various tissue-specific sources of MSCs [44-46]. GO analysis identified several cellular processes (TLR signaling, mitotic cell cycle, post-translation protein modification, extracellular matrix disassembly, apoptotic signaling pathway) that are vital in BMSC survival, proliferation, migration, and differentiation.

SDF-1 signaling has been recognized for its chemotactic and osteogenic function in BMSCs. Our findings further solidify its expanded role in BMSC biology. 
We have elucidated several miRNAs that are upregulated and downregulated in response to SDF-1. Among these miRNAs, we correlated some of their functions with roles identified in existing literature, and we characterized novel miRNAs concerning their potential targets in BMSCs (Table 2). We also determined key signaling pathways that are collectively influenced by the differentially expressed miRNAs. Limitations to our study do exist. Our study only utilized BMSCs in tissue culture and thus needs to be validated in vivo. Moreover, our study only utilized one concentration and one time period for SDF-1 treatment. It is valuable to understand the dose and time-dependent effects of SDF-1 in vitro and in vivo models. Overall, our study indicated that SDF-1 induces some of its osteogenic and chemotactic effects by regulating miRNAs in human BMSCs.

Funding: This publication is based upon work supported in part by the National Institutes of Health AG036675 (National Institute on Aging-AG036675 S.F, W.D.H, M.H, C.S,). The funding mentioned above did not lead to any conflict of interest regarding the publication of this manuscript.

Conflict of Interest: Authors state no conflict of interest.

Data Availability Statement: The datasets generated during and/or analysed during the current study are available from the corresponding author on reasonable request.

\section{References}

1. Sangani R, Periyasamy-Thandavan S, Kolhe R, Bhattacharyya MH, Chutkan N, Hunter M, et al. MicroRNAs-141 and 200a regulate the SVCT2 transporter in bone marrow stromal cells. Mol Cell Endocrinol. 2015 Jul;410:19-26.

2. Pittenger MF, Mackay AM, Beck SC, Jaiswal RK, Douglas R, Mosca JD, et al. Multilineage potential of adult human mesenchymal stem cells. Science. 1999 Apr;284(5411):143-7.

3. Jones DL, Wagers AJ. No place like home: anatomy and function of the stem cell niche. Nat Rev Mol Cell Biol. 2008 Jan;9(1):11-21.

4. Gong J, Meng HB, Hua J, Song ZS, He ZG, Zhou B, et al. The SDF-1/CXCR4 axis regulates migration of transplanted bone marrow mesenchymal stem cells towards the pancreas in rats with acute pancreatitis. Mol Med Rep. 2014 May;9(5):1575-82.

5. Yu L, Cecil J, Peng SB, Schrementi J, Kovacevic S, Paul D, et al. Identification and expression of novel isoforms of human stromal cell-derived factor 1. Gene. 2006 Jun;374:174-9.

6. Guang LG, Boskey AL, Zhu W. Age-related CXC chemokine receptor-4-deficiency impairs osteogenic differentiation potency of mouse bone marrow mesenchymal stromal stem cells. Int J Biochem Cell Biol. 2013 Aug;45(8):1813-20.

7. Bobis-Wozowicz S, Miekus K, Wybieralska E, Jarocha D, Zawisz A, Madeja Z, et al. Genetically modified adipose tissue-derived mesenchymal stem cells overexpressing CXCR4 display increased motility, invasiveness, and homing to bone marrow of NOD/SCID mice. Exp Hematol. 2011 Jun;39(6):686-696.e4.

8. Herberg S, Aguilar-Perez A, Howie RN, Kondrikova G, PeriyasamyThandavan S, Elsalanty ME, et al. Mesenchymal stem cell expression of SDF-1 $\beta$ synergizes with BMP-2 to augment cellmediated healing of critical-sized mouse calvarial defects. I Tissue Eng Regen Med. 2017 Jun;11(6):1806-19.

9. Herberg S, Shi X, Johnson MH, Hamrick MW, Isales CM, Hill WD. Stromal cell-derived factor- $1 \beta$ mediates cell survival through enhancing autophagy in bone marrow-derived mesenchymal stem cells. PLoS One. 2013;8(3):e58207.

10. Herberg S, Fulzele S, Yang N, Shi X, Hess M, PeriyasamyThandavan S, et al. Stromal cell-derived factor-1 $\beta$ potentiates bone morphogenetic protein-2-stimulated osteoinduction of genetically engineered bone marrow-derived mesenchymal stem cells in vitro. Tissue Eng Part A. 2013 Jan;19(1-2):1-13.

11. Bragg R, Gilbert W, Elmansi AM, Isales CM, Hamrick MW, Hill WD, et al. Stromal cell-derived factor-1 as a potential therapeutic target for osteoarthritis and rheumatoid arthritis. Ther Adv Chronic Dis. 2019 Oct;10:2040622319882531.

12. Gilbert W, Bragg R, Elmansi AM, McGee-Lawrence ME, Isales CM, Hamrick MW, et al. Stromal cell-derived factor-1 (CXCL12) and its role in bone and muscle biology. Cytokine. 2019 Nov;123:154783.

13. Granero-Moltó F, Weis JA, Miga MI, Landis B, Myers TJ, O国ear $L$, et al. Regenerative effects of transplanted mesenchymal stem cells in fracture healing. Stem Cells. 2009 Aug;27(8):1887日98.

14. Liu X, Zhou C, Li Y, Ji Y, Xu G, Wang X, et al. SDF-1 promotes endochondral bone repair during fracture healing at the traumatic brain injury condition. PLoS One. 2013;8(1):e54077.

15. Kitaori T, Ito H, Schwarz EM, Tsutsumi R, Yoshitomi H, Oishi S, et al. Stromal cell-derived factor $1 /$ CXCR4 signaling is critical for the recruitment of mesenchymal stem cells to the fracture site during skeletal repair in a mouse model. Arthritis Rheum. 2009 Mar;60(3):813-23.

16. Periyasamy-Thandavan S., Burke J., Mendhe B., Kondrikova G., Kolhe R., Hunter M., et al. MicroRNA-141-3p Negatively Modulates SDF-1 Expression in Age-Dependent Pathophysiology of Human and Murine Bone Marrow Stromal Cells. J Gerontol A Biol Sci Med Sci. 2019 Aug 16;74(9):1368-1374.

17. Weinhold B. Epigenetics: the science of change. Environ Health Perspect. 2006 Mar;114(3):A160-7.

18. Yannarelli G, Pacienza N, Cuniberti L, Medin J, Davies J, Keating A. Brief report: the potential role of epigenetics on multipotent cell differentiation capacity of mesenchymal stromal cells. Stem Cells. 2013 Jan;31(1):215-20.

19. Challen GA, Sun D, Jeong M, Luo M, Jelinek J, Berg JS, et al. Dnmt3a is essential for hematopoietic stem cell differentiation. Nat Genet. 2011 Dec;44(1):23-31.

20. Arnsdorf EJ, Tummala P, Castillo AB, Zhang F, Jacobs CR. The epigenetic mechanism of mechanically induced osteogenic differentiation. J Biomech. 2010 Nov;43(15):2881-6.

21. Alexanian AR. Epigenetic modifiers promote efficient generation of neural-like cells from bone marrow-derived mesenchymal 
cells grown in neural environment. J Cell Biochem. 2007 Feb;100(2):362-71.

22. Fu G, Ren A, Qiu Y, Zhang Y. Epigenetic Regulation of Osteogenic Differentiation of Mesenchymal Stem Cells. Curr Stem Cell Res Ther. 2016;11(3):235-46.

23. He L, Hannon GJ. MicroRNAs: small RNAs with a big role in gene regulation. Nat Rev Genet. 2004 Jul;5(7):522-31.

24. Nicolas FE, Lopez-Martinez AF. MicroRNAs in human diseases. Recent Pat DNA Gene Seq. 2010 Nov;4(3):142-54.

25. Yi R, Fuchs E. MicroRNAs and their roles in mammalian stem cells. J Cell Sci. 2011 Jun;124(Pt 11):1775-83.

26. Luo W, Nie Q, Zhang X. MicroRNAs involved in skeletal muscle differentiation. J Genet Genomics. 2013 Mar;40(3):107-16.

27. Mathieu J, Ruohola-Baker H. Regulation of stem cell populations by microRNAs. Adv Exp Med Biol. 2013;786:329-51.

28. Cruz-Santos MC, Aragón-Raygoza A, Espinal-Centeno A, Arteaga-Vázquez M, Cruz-Hernández A, Bako L, et al. The Role of microRNAs in Animal Cell Reprogramming. Stem Cells Dev. 2016 Jul;25(14):1035国49.

29. Clough E, Barrett T. The Gene Expression Omnibus Database. Methods Mol Biol. 2016;1418:93-110.

30. Hwang HD, Lee JT, Koh JT, Jung HM, Lee HJ, Kwon TG. Sequential Treatment with SDF-1 and BMP-2 Potentiates Bone Formation in Calvarial Defects. Tissue Eng Part A. 2015 Jul;21(13-14):2125-35.

31. Shen X, Zhang Y, Gu Y, Xu Y, Liu Y, Li B, et al. Sequential and sustained release of SDF-1 and BMP-2 from silk fibroinnanohydroxyapatite scaffold for the enhancement of bone regeneration. Biomaterials. 2016 Nov;106:205-16.

32. Beederman M, Lamplot JD, Nan G, Wang J, Liu X, Yin L, et al. BMP signaling in mesenchymal stem cell differentiation and bone formation. J Biomed Sci Eng. 2013 Aug;6(8 8A):32-52.

33. Marupanthorn K, Tantrawatpan C, Kheolamai P, Tantikanlayaporn $\mathrm{D}$, Manochantr S. Bone morphogenetic protein-2 enhances the osteogenic differentiation capacity of mesenchymal stromal cells derived from human bone marrow and umbilical cord. Int J Mol Med. 2017 Mar;39(3):654-62.

34. Sun J, Li J, Li C, Yu Y. Role of bone morphogenetic protein-2 in osteogenic differentiation of mesenchymal stem cells. Mol Med Rep. 2015 Sep;12(3):4230-7.

35. Wei JQ, Chen H, Zheng XF, Zhang BX, Wang Y, Tang PF, et al. [Hsa-miR-654-5p regulates osteogenic differentiation of human bone marrow mesenchymal stem cells by repressing bone morphogenetic protein 2]. Nan Fang Yi Ke Da Xue Xue Bao. 2012 Mar;32(3):291-5.

36. Chen H, Ji X, She F, Gao Y, Tang P. miR-628-3p regulates osteoblast differentiation by targeting RUNX2: possible role in atrophic non-union. Int J Mol Med. 2017 Feb;39(2):279-86.

37. Lykissas $M$, Gkiatas I. Use of recombinant human bone morphogenetic protein-2 in spine surgery. World J Orthop. 2017 Jul;8(7):531-5.

38. Zhou J, Nie H, Liu P, Wang Z, Yao B, Yang L. Down-regulation of miR-339 promotes differentiation of BMSCs and alleviates osteoporosis by targeting DLX5. Eur Rev Med Pharmacol Sci. 2019 Jan;23(1):29-36.

39. Sun Y, Xu J, Xu L, Zhang J, Chan K, Pan X, et al. MiR-503 Promotes Bone Formation in Distraction Osteogenesis through Suppressing Smurf1 Expression. Sci Rep. 2017 Mar;7(1):409.
40. Han L, Zhou Y, Zhang R, Wu K, Lu Y, Li Y, et al. MicroRNA Let7f-5p Promotes Bone Marrow Mesenchymal Stem Cells Survival by Targeting Caspase-3 in Alzheimer Disease Model. Front Neurosci. 2018 May;12:333.

41. Tie Y, Chen C, Yang Y, Qian Z, Yuan H, Wang H, et al. Upregulation of let-7f-5p promotes chemotherapeutic resistance in colorectal cancer by directly repressing several pro-apoptotic proteins. Oncol Lett. 2018 Jun;15(6):8695-702.

42. Rashid MA, Haque M, Akbar M. Role of Polyunsaturated Fatty Acids and Their Metabolites on Stem Cell Proliferation and Differentiation. Adv Neurobiol. 2016;12:367-80.

43. Fillmore N, Huqi A, Jaswal JS, Mori J, Paulin R, Haromy A, et al. Effect of fatty acids on human bone marrow mesenchymal stem cell energy metabolism and survival. PLoS One. 2015 Mar;10(3):e0120257.

44. Kang JX, Wan JB, He C. Concise review: regulation of stem cell proliferation and differentiation by essential fatty acids and their metabolites. Stem Cells. 2014 May;32(5):1092-8.

45. Levental KR, Surma MA, Skinkle AD, Lorent JH, Zhou Y, Klose C, et al. $\omega-3$ polyunsaturated fatty acids direct differentiation of the membrane phenotype in mesenchymal stem cells to potentiate osteogenesis. Sci Adv. 2017 Nov;3(11):eaa01193. https://doi. org/10.1126/sciadv.aao1193.

46. Das UN. Influence of polyunsaturated fatty acids and their metabolites on stem cell biology. Nutrition. 2011 Jan;27(1):21-5. 